\title{
Revision Total Knee Arthroplasty with Metaphyseal Sleeves without Stem: Short-Term Results
}

\author{
Giacomo Stefani $^{1}$ Valerio Mattiuzzo ${ }^{1}$ Greta Prestini ${ }^{1}$ \\ 1 Dipartimento Di Ortopedia 2, Istituto Clinico "Città di Brescia," \\ Brescia, Italy \\ Address for correspondence Giacomo Stefani, MD, Dipartimento Di \\ Ortopedia 2, Istituto Clinico Città di Brescia, Via Gualla 15, Brescia \\ 25128, Italy (e-mail: trstefa@libero.it).
}

Joints 2017;5:207-211.

\begin{abstract}
Purpose The aim of this study was to evaluate the efficacy of revision total knee arthroplasty (TKA) with cementless metaphyseal sleeves without stems either in the femoral or tibial side or in both.

Methods In this retrospective study, 51 patients ( 51 knees) operated in the period 2010 to 2015 met the above-mentioned criteria and were invited to a medical examination including X-rays. Forty-six were available for the study. Mean follow-up was 37 months. Knee Society score (KSS) (objective knee score), Western Ontario and McMaster Universities Osteoarthritis Index (WOMAC) score, and range of motion (ROM) were used as outcome scores and compared with baseline values. X-rays were also examined and compared with postoperative exams to evaluate the presence of loosening, radiolucent lines, and bone ingrowth. Satisfaction of the patients was also investigated using a linear scale from 1 to 10 .

Results KSS improved from 39 to $77(p<0.01)$; WOMAC score improved from 76 to $41(p<0.01)$. Twenty-four $(52 \%)$ patients were satisfied, 15 (32\%) were partially satisfied, and $7(16 \%)$ were unsatisfied. ROM improved from 93 to 96 degrees

Keywords

- knee

- total knee arthroplasty

- revision

- methaphyseal sleeves

- results (nonsignificant difference). X-rays showed no loosening of the implants, radiolucent lines in 4 patients ( 3 of them were asymptomatic) and bone ingrowth in 43 out of 46 patients.

Conclusion In this short-term retrospective study, the use of sleeves without stem was a safe and effective procedure in revision TKA. We found a significant improvement in clinical results compared with baseline values and no signs of implant loosening. Level of Evidence Level IV, therapeutic case series.
\end{abstract}

\section{Introduction}

Total knee arthroplasty (TKA) is a highly successful surgical procedure in the treatment of degenerative and inflammatory disease of the knee joint, ${ }^{1}$ and in the past decades, it has also become very popular due to the improvement in materials and design. In addition, survivorship of the implants increased, as described in the literature, and this led to increasing volumes of total joint replacements. ${ }^{2-4}$

Nevertheless, TKA may fail and this can happen through a variety of mechanism including wear, loosening, infection, instability, stiffness, and fracture. ${ }^{5}$ Failure of TKA requires a revision surgery that is often a complex surgical procedure, with results far less satisfactory than primary implants. ${ }^{6}$ Increasing volume of primary TKAs is projecting the number of revisions to reach 268,200 by the year 2030 only in the United States. ${ }^{7}$

Usually bone deficiency and implant fixation are the most critical problems in revision $\mathrm{TKA}^{8}$ and pose the questions of finding a sound fixation of the new implant and management of bone loss. So far, fixation has been achieved with intramedullary stems either cemented or press fit, with satisfactory results, albeit a few problems have been reported such as pain at the tip of the stem and difficulties in finding a correct position of the stem itself if tibia and femoral canals are not straight, with the need of offset devices. ${ }^{9-11}$
DOI https://doi.org/ 10.1055/s-0037-1607427. ISSN 2282-4324.
Copyright (c) 2017 Georg Thieme Verlag License terms KG Stuttgart - New York 


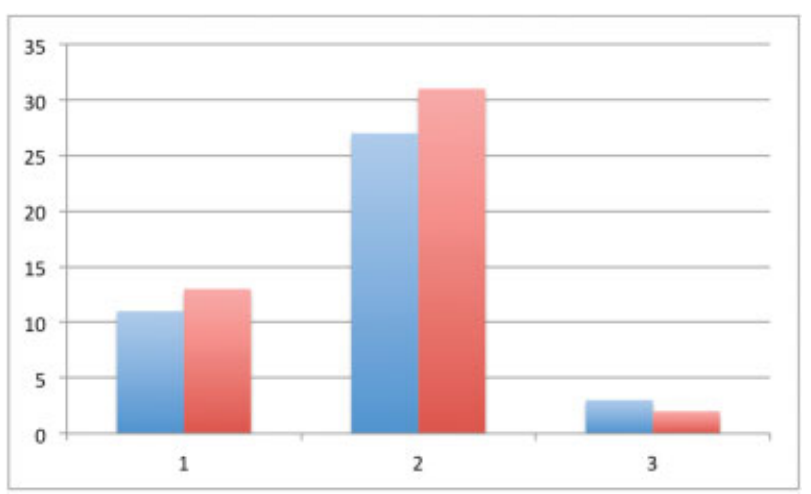

Fig. 1 AORI classification of bone defects in the femurs (blue) and tibias (red), at the time of revision surgery (number of cases on the $y$-axis; AORI types on the $x$-axis). AORI, Anderson Orthopaedic Research Institute.

Bone deficiency has been managed in different ways such as cement, bone chips, or structural bone allografts, but the latter requires a technically demanding surgical procedure, with grafts not easily available in most centers and with problems due to resorption of graft itself and possible disease transmission. Porous tantalum cones are effectively used for management of bone loss, but they have two interfaces, one with bone and one with implant, and this may be a weak link of the implant. $^{12-17}$

In the past years, metaphyseal fixation has been considered as a valid option to improve construct stability while managing bone loss at the same time. ${ }^{18}$ Quite a few authors have been using cementless metaphyseal sleeves in revision cases as support of stems, reporting satisfactory midterm results. ${ }^{19-21}$ Indeed, pain at the tip of the stem after revision TKA has been widely reported, ${ }^{22}$ and the use of metaphyseal sleeves alone may be an effective alternative to stem fixation.
The purpose of this study was to assess efficacy of metaphyseal sleeves without stem in revision TKAs. The hypothesis of the study was that metaphyseal sleeves without stem might be an effective option for both implant fixation and management of bone loss in revision TKAs.

\section{Methods}

\section{Study Population}

From January 2010 to December 2015, 51 patients underwent revision TKA with the use of cementless metaphyseal sleeves without stem either on the tibia or femoral side or in both. Thirty-six (71\%) patients were female and 15 (29\%) were male. Mean age at the time of revision was 71 years (range, 5287 years). In all cases, we used the SIGMA TC3 implant (DePuy Synthes, Raynham, Massachusetts, United States). Forty-one femoral and 46 tibial metaphyseal sleeves were used.

Reasons for revisions were aseptic loosening in 26 (51\%) cases, periprosthetic joint infection in 17 (33\%), stiffness in 3 (6\%), polyethylene wear in $3(6 \%)$, fracture in $1(2 \%)$, and implant failure in 1 (2\%). A first revision was performed in 39 (76\%) patients, second revision in 10 (20\%), and third revision in 2 (4\%). Bone defects prior to revision surgery were classified according to Anderson Orthopaedic Research Institute classification. There was a type 3 defect in 3 femurs and 2 tibias, types $2 \mathrm{~A}$ and $2 \mathrm{~B}$ in 27 femurs and 31 tibias, and type 1 in 11 femurs and 13 tibias (-Fig. 1 ).

\section{Intervention}

After arthrotomy, components of the failed TKA were extracted. Nineteen (38\%) patients (17 in the infection group and 2 in the stiffness group) had an anterior tibial tubercle (ATT) osteotomy during the surgical approach that was fixed with either screws, metal wire, or both (-Fig. 2). We had three
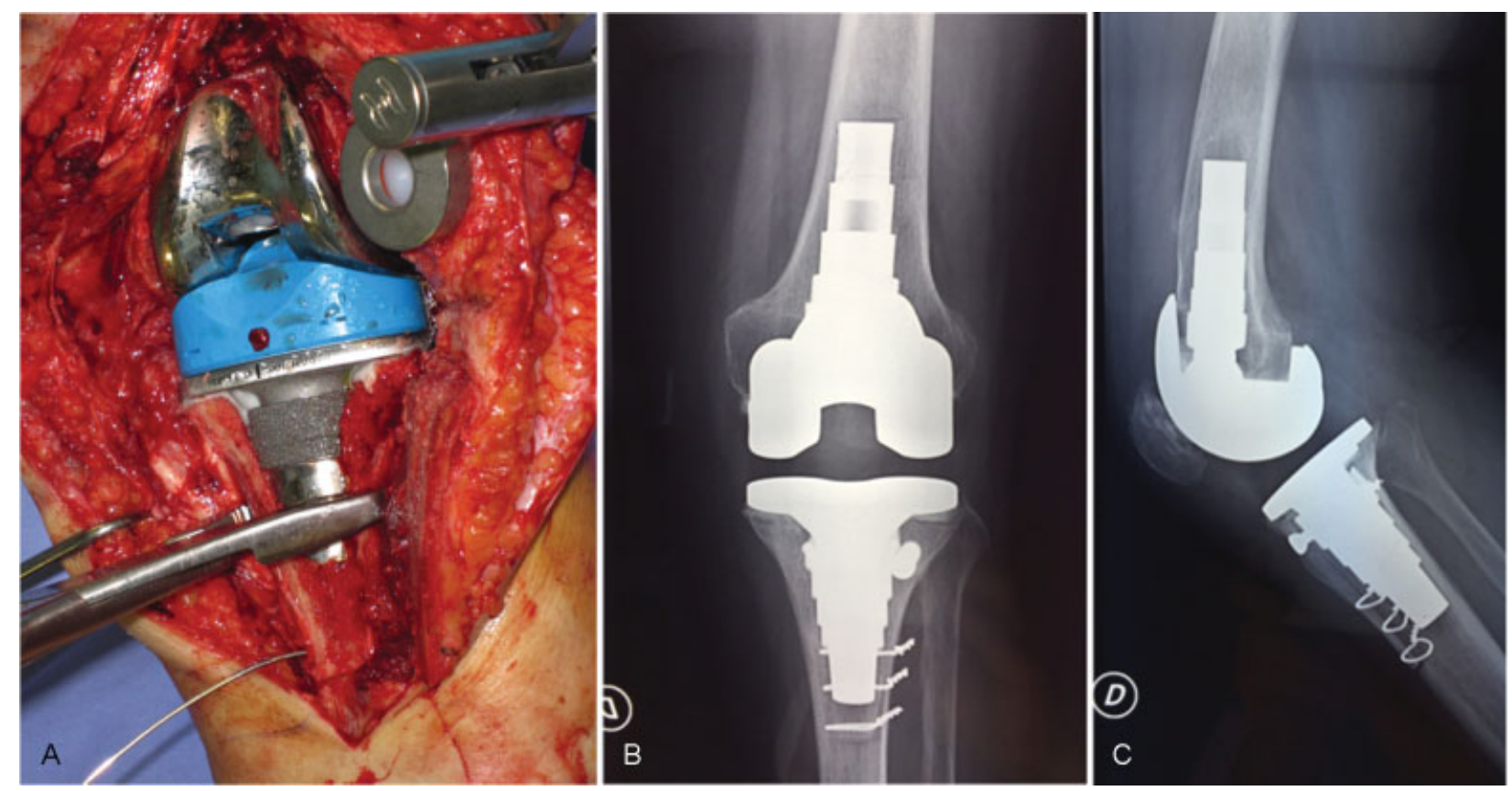

Fig. 2 Revision total knee arthroplasty for periprosthetic joint infection. (A) Osteotomy of anterior tibial tuberosity did not impair sleeve and implant stability. (B, C) Postoperative radiographs. Fixation of the tibial tuberosity can be achieved with metal wires, screws, or both. 
linear anterior tibia fractures going down into the diaphysis up to $3 \mathrm{~cm}$. The tibial canal was then identified and reamed to 10 to $12 \mathrm{~mm}$ in width and approximately $150 \mathrm{~mm}$ in depth using a conical reamer. Preparation of the tibia for the sleeve was done using sequential broaching with broaches with a narrow stem at their tip to maintain the correct direction in the canal. Broaching depth was achieved at the level that we consider optimal for obtaining a correct joint line, according to preoperative planning. Fixation of the broach was considered as acceptable when it is possible to lift the leg off the table using the broaching jig. We then cut proximal tibia and quite often, this was done using the broach itself as a support base for cutting. We then measured extension and flexion gap with spacers and proceeded to femur preparation in a similar way. Once we found the correct direction of the broach, we removed the guide stem, this allowed slight flexion of the broach to compensate the flexion gap (when necessary), and then we kept broaching until we obtained a good stability. Stability in the femur was checked by rotating the handle of the broach and observing the rotation of the femur as well. Position of the tibia tray was obtained by keeping the center of the tibial tubercle as reference and femoral component with the balance technique at 90 degrees of flexion. Fixation of the final components with sleeves was done with cement on the baseplate only, being careful to avoid putting cement on the sleeve. Cement with antibiotics was used in all cases. A posterior-stabilized insert was used in 47 patients and a TC3 (VVC constraint) insert in 4 cases. No hinged inserts were used.

Weight bearing was allowed as tolerated and encouraged to reach a good range of motion (ROM) since the first postoperative day. Weight bearing as tolerated was allowed also in patients with an ATT osteotomy with a brace locked in extension for 6 weeks. Continuous passive motion up to 90 degrees was used for 30 days after surgery.

\section{Outcome Measurements}

Patients were evaluated before surgery and at follow-up according to the objective Knee Society score (KSS) and the Western Ontario and McMaster Universities Osteoarthritis Index (WOMAC) score (function and ROM). A satisfaction score was also obtained on a linear scale from 1 to 10 and patients rated as satisfied (score 7-10), partially satisfied (4-6), and unsatisfied (1-3). Plain radiographs were also taken at follow-up and compared with postoperative radiographs to evaluate bone ingrowth and the presence of radiolucent lines.

\section{Data Analysis}

A paired Student's $t$-test was used for statistical analysis to compare outcome scores with baseline values. Significance was set for $p$-value $<0.05$.

\section{Results}

Out of 51, 46 patients were available for the study. Reasons for exclusion were death, ${ }^{1}$ new revision for infection, ${ }^{1}$ loss of patient traceability, ${ }^{2}$ and refusal to enter the study. ${ }^{1}$ Average follow-up was 37 months (range, 14-58 months).
The mean KSS score improved from 39 at baseline to 77 at follow-up ( $p<0.01$ ), and mean WOMAC score improved from 76 at baseline to 41 follow-up ( $p<0.01$ ). No significant improvement in ROM was observed from baseline to follow-up (93 and 96 degrees, respectively). Twenty-four (52\%) patients were satisfied with the result, 15 (32\%) were partially satisfied, and 7 (16\%) were unsatisfied. Among these, six patients belonged to the infection group and one to the stiffness group that did not improved in ROM after surgery.

X-rays showed an overall good fixation of the implants. Bone ingrowth was seen in 42 out of 46 knees ( - Fig. 3). In four patients, radiolucent lines (three femoral and one tibial) were seen around the sleeves, three of those without clinical symptoms. Radiological cortical contact of the sleeves was found in 12 patients (seven in the femur, on the anterior cortex, and five in the tibia) but no pain was related to that. All the ATT osteotomies showed good fixation without secondary displacement of the tibial tubercle.

\section{Discussion}

The purpose of this study was to evaluate the clinical efficacy of revision TKA with cementless metaphyseal sleeves used for implant fixation without stems in an attempt to improve construct stability while managing bone deficiency. Although this fixation was initially considered as a support to diaphyseal fixation, it was recently suggested as an alternative to it. ${ }^{18}$

Indeed, stem fixation has been used for a long time in revision TKA with satisfactory results. However, some problems have been described such as pain at the tip of the stem ${ }^{22}$ and difficulties in positioning the stem itself if the femoral and tibia canal were not straight, ${ }^{9-11}$ even with off-set design, thus impairing the efficacy of the treatment. Moreover, in many cases, the metaphyseal region has still fairly good bone quality and it seems reasonable to use it to fix the implant and not to bypass it with a stem. This hypothesis was confirmed by the results of this study. Radiographs at follow-up showed an overall stable fixation of the implants without signs of loosening. Radiological bone ingrowth was seen in 42 out of 46 knees, similarly to what reported by Dalury and Barret. ${ }^{19}$ Moreover, clinical evaluation showed significant improvement of mean KSS and WOMAC scores and patients' overall satisfaction (satisfied or partially satisfied) was reported in 39 (84\%) patients, thus confirming the efficacy of the procedure.

The use of sleeves is getting now popular and some authors showed satisfactory results in revision TKA. ${ }^{19-21}$ Although sleeves are usually used with stems, Bugler et $\mathrm{al}^{20}$ reported on implants without stems in a percentage of cases, both in the tibia and in the femur, and showed no evidence of early loosening, thus confirming the efficacy of this treatment.

According to zonal fixation concept, two zones should be required for a stable fixation in revision TKA. ${ }^{23}$ Although this is mandatory with the use of stems, sleeves provide good fixation in a large area in zone 2 (metaphysis), thus making fixation in zone 3 (diaphysis) less relevant. Therefore, stem size and percentage of canal filling can be reduced, ${ }^{21}$ and the use of stem can be even avoided. Moreover, stemless femoral component can be easily flexed to compensate flexion gap 

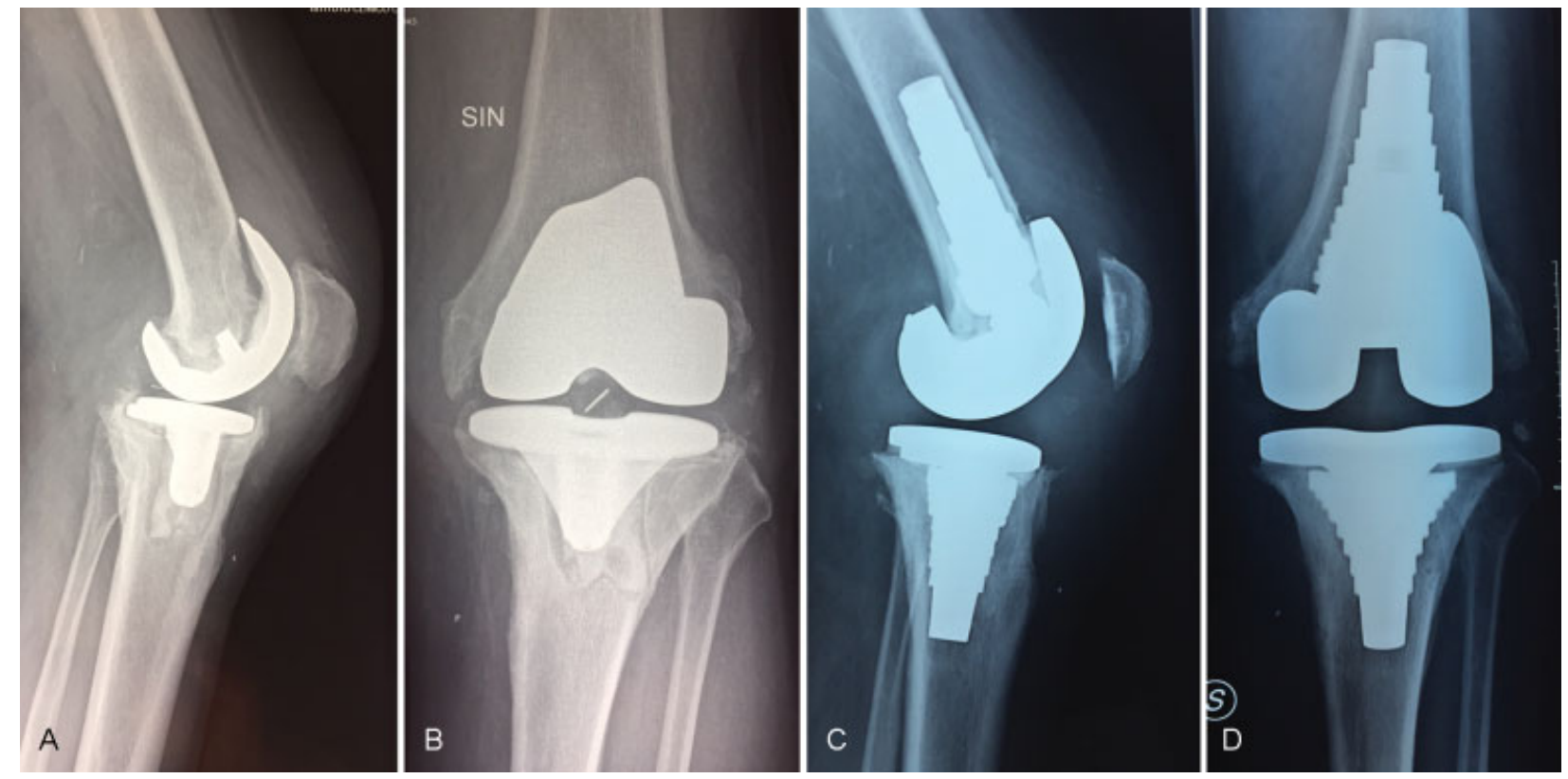

Fig. 3 (A, B) Aseptic loosening of a total knee arthroplasty. Note the important bone loss both on distal femur and proximal tibia. A lytic area in the cortical bone of the lateral metaphyseal region of the tibia is evident. (C, D) Revision TKA of the same case at 33-month follow-up. Note the bone ingrowth in the lytic area.

(larger than extension gap in most cases), if necessary. In fact, stemless femoral component can be flexed up to 6 or 7 degrees, thus filling up to $5 \mathrm{~mm}$ of flexion gap.

We observed that in some cases, the proximal part of the sleeve was in contact with the anterior cortex of the femur, albeit this finding was not related to pain. Probably, sleeve is mostly loaded on its proximal part, which is far from the contact area.

Nevertheless, stems are necessary during surgery to find a correct alignment in the medullary canal and we believe that a narrow stem is acceptable to maintain a straight direction and correct placement of the implant; however, stems should have no contact with diaphyseal cortical bone.

In all the septic revision cases and in two of the stiff group, we performed an ATT osteotomy. In all these cases, we were able, despite the sleeve, to obtain a stable fixation of the tuberosity. In three cases of this group, we also had a vertical narrow tibia fracture going down in the diaphysis for 2 to $3 \mathrm{~cm}$ while positioning the sleeve. These fractures did not require modification of implant position.

In this study, ROM assessment showed a very limited improvement after surgery (93-96 degrees). Graichen et $\mathrm{al}^{21}$ excluded septic revision from their study and showed a significant improvement in ROM. Conversely, Dalury and Barret ${ }^{19}$ showed worsening of ROM from 125 degrees preoperative to 115 degrees at final follow-up. In our opinion, limited increase in ROM depends on the fact that we included a large number of septic revisions (38\%) in which we used a brace and allowed a limited ROM during the initial rehabilitation period, thus obtaining a limited final ROM.

Another potential advantage of sleeve fixation is that sleeves are much closer to the joint line than stems, thus improving ligament balance ${ }^{24}$ and allowing use of unconstrained implants that may have longer survivorship. ${ }^{25}$
As a final consideration, the use of sleeves without stems is a money saving procedure. On considering the economic impact of revision TKA in health care, the use of less expensive procedures, once their efficacy is confirmed, is desirable.

There are few limitations in our study. This is a retrospective evaluation of a nonconsecutive case series, which implies a risk of selection bias. Moreover, no control group was available. The short follow-up time is another limitation. Although some studies ${ }^{26-28}$ showed that at least $50 \%$ of all rerevision occur in the first 2 to 3 years, we are aware that longer follow-up is required to validate this procedure.

In conclusion, in this short-term retrospective study, the use of sleeves without stem was a safe and effective procedure in revision TKA. We found a significant improvement in clinical results compared with baseline values and no signs of implant loosening.

\section{Conflict of Interest}

One of the authors, G.S., is a paid consultant of DePuy Synthes.

\section{References}

1 Ethgen O, Bruyère O, Richy F, Dardennes C, Reginster JY. Healthrelated quality of life in total hip and total knee arthroplasty. A qualitative and systematic review of the literature. J Bone Joint Surg Am 2004;86-A(05):963-974

2 Rodriguez JA, Bhende H, Ranawat CS. Total condylar knee replacement: a 20-year followup study. Clin Orthop Relat Res 2001; (388):10-17

3 Schai PA, Thornhill TS, Scott RD. Total knee arthroplasty with the PFC system. Results at a minimum of ten years and survivorship analysis. J Bone Joint Surg Br 1998;80(05):850-858 
4 Scuderi GR, Insall JN, Windsor RE, Moran MC. Survivorship of cemented knee replacements. J Bone Joint Surg Br 1989;71(05): 798-803

5 Rand JA, Trousdale RT, Ilstrup DM, Harmsen WS. Factors affecting the durability of primary total knee prostheses. J Bone Joint Surg Am 2003;85-A(02):259-265

6 Swedish Knee Arthroplasty Register. Annual Report 2014. Available at: http://www.myknee.se/pdf/SKAR2014_Eng_1.1.pdf

7 Kurtz S, Ong K, Lau E, Mowat F, Halpern M. Projections of primary and revision hip and knee arthroplasty in the United States from 2005 to 2030. J Bone Joint Surg Am 2007;89(04):780-785

8 Engh GA, Ammeen DJ. Bone loss with revision total knee arthroplasty: defect classification and alternatives for reconstruction. Instr Course Lect 1999;48:167-175

9 Fehring TK, Odum S, Olekson C, Griffin WL, Mason JB, McCoy TH. Stem fixation in revision total knee arthroplasty: a comparative analysis. Clin Orthop Relat Res 2003;(416):217-224

10 Whaley AL, Trousdale RT, Rand JA, Hanssen AD. Cemented longstem revision total knee arthroplasty. J Arthroplasty 2003;18(05): 592-599

11 Manopoulos P, Havet E, Pearce O, Lardanchet JF, Mertl P. Mid- to long-term results of revision total knee replacement using pressfit intramedullary stems with cemented femoral and tibial components. J Bone Joint Surg Br 2012;94(07):937-940

12 Whiteside LA. Morselized allografting in revision total knee arthroplasty. Orthopedics 1998;21(09):1041-1043

13 Dorr LD, Ranawat CS, Sculco TA, et al. Bone graft for tibial defects in total knee arthroplasty. Clin Orthop Relat Res 1986;(205): 153-165

14 Bauman RD, Lewallen DG, Hanssen AD. Limitations of structural allograft in revision total knee arthroplasty. Clin Orthop Relat Res 2009;467(03):818-824

15 Sculco PK, Abdel MP, Hanssen AD, Lewallen DG. The management of bone loss in revision total knee arthroplasty: rebuild, reinforce, and augment. Bone Joint J 2016;98-B(1, suppl A):120-124

16 Meneghini RM, Lewallen DG, Hanssen AD. Use of porous tantalum metaphyseal cones for severe tibial bone loss during revision total knee replacement. J Bone Joint Surg Am 2008;90(01):78-84
17 Kamath AF, Lewallen DG, Hanssen AD. Porous tantalum metaphyseal cones for severe tibial bone loss in revision knee arthroplasty: a five to nine-year follow-up. J Bone Joint Surg Am 2015;97 (03):216-223

18 Haidukewych GJ, Hanssen A, Jones RD. Metaphyseal fixation in revision total knee arthroplasty: indications and techniques. J Am Acad Orthop Surg 2011;19(06):311-318

19 Dalury DF, Barrett WP. The use of metaphyseal sleeves in revision total knee arthroplasty. Knee 2016;23(03):545-548

20 Bugler KE, Maheshwari R, Ahmed I, Brenkel IJ, Walmsley PJ. Metaphyseal sleeves for revision total knee arthroplasty: good short-term outcomes. J Arthroplasty 2015;30(11):1990-1994

21 Graichen H, Scior W, Strauch M. Direct, cementless, methaphyseal fixation in knee revision arthroplasty with sleeves: short-term results. J Arthroplasty 2015;30(12):2256-2259

22 Barrack RL, Rorabeck C, Burt M, Sawhney J. Pain at the end of the stem after revision total knee arthroplasty. Clin Orthop Relat Res 1999;(367):216-225

23 Morgan-Jones R, Oussedik SI, Graichen H, Haddad FS. Zonal fixation in revision total knee arthroplasty. Bone Joint J 2015; 97-B(02):147-149

24 Bieger R, Huch K, Kocak S, Jung S, Reichel H, Kappe T. The influence of joint line restoration on the results of revision total knee arthroplasty: comparison between distance and ratio-methods. Arch Orthop Trauma Surg 2014;134(04):537-541

25 Shen C, Lichstein PM, Austin MS, Sharkey PF, Parvizi J. Revision knee arthroplasty for bone loss: choosing the right degree of constraint. J Arthroplasty 2014;29(01):127-131

26 Mortazavi SM, Molligan J, Austin MS, Purtill JJ, Hozack WJ, Parvizi J. Failure following revision total knee arthroplasty: infection is the major cause. Int Orthop 2011;35(08):1157-1164

27 Ghomrawi HMK, Kane RL, Eberly LE, Bershadsky B, Saleh KJ; North American Knee Arthroplasty Revision (NAKAR) Study Group. Patterns of functional improvement after revision knee arthroplasty. J Bone Joint Surg Am 2009;91(12):2838-2845

28 Schroer WC, Berend KR, Lombardi AV, et al. Why are total knees failing today? Etiology of total knee revision in 2010 and 2011. J Arthroplasty 2013;28(8, Suppl):116-119 